\title{
Detection of extended spectrum $\beta$-lactamase in Pseudomonas spp. isolated from two tertiary care hospitals in Bangladesh
}

\author{
Shahanara Begum ${ }^{1}$, Md Abdus Salam², Kh Faisal Alam², Nurjahan Begum³ ${ }^{2}$ Pervez Hassan ${ }^{4}$
} and Jalaluddin Ashraful $\mathrm{Haq}^{5}$

\begin{abstract}
Background: Extended spectrum ß-lactamases (ESBLS) represent a major group of lactamases responsible for resistance, mostly produced by gram-negative bacteria, to newer generations of ß-lactam drugs currently being identified in large numbers worldwide. The present study was undertaken to see the frequency of ESBL producing Pseudomonas spp. isolated from six hundred clinical specimens (wound, pus, aural, urine, sputum, throat and other swabs) collected over a period of three years from two tertiary care hospitals in Bangladesh.

Findings: Aerobic bacterial culture was performed on aseptically collected swabs and only growth of Pseudomonas was considered for further species identification and ESBL production along with serotyping of Pseudomonas aeruginosa. Antimicrobial susceptibility testing was carried out using the Kirby-Bauer agar diffusion method and ESBL production was detected on Mueller Hinton agar by double-disk synergy technique using Amoxicillin-Clavulanic acid with Ceftazidime, Cefotaxime, Ceftriaxone and Aztreonam. Culture yielded 120 Pseudomonas spp. and 82 of them were biochemically characterized for species. Pseudomonas aeruginosa was found to be the predominant (90.2\%) species. Of 82 isolates tested for ESBL, 31 (37.8\%) were ESBL positive with 29 (93.5\%) as Pseudomonas aeruginosa, the remaining 2 (6.5\%) were Stenotrophomonas maltophilia and Ralstonia pickettii. Antibiogram revealed Imipenem as the most effective drug (93.3\%) among all antimicrobials used against Pseudomonas spp. followed by Aminoglycosides (63.7\%).
\end{abstract}

Conclusion: ESBL producing Pseudomonas spp. was found to be a frequent isolate from two tertiary care hospitals in Bangladesh, showing limited susceptibility to antimicrobials and decreased susceptibility to Imipenem in particular, which is a matter of great concern.

Keywords: Pseudomonas spp, Antimicrobial susceptibility, ESBL

\section{Background}

The worldwide emergence of multi-drug resistant bacterial strains is a growing concern, especially infections caused by Pseudomonas spp. and $P$. aeruginosa in particular. $P$. aeruginosa is an opportunistic pathogen with innate resistance to many antibiotics and disinfectants including anti-pseudomonal Penicillins, Ceftazidime, Carbapenems, Aminoglycosides and Ciprofloxacin [1]. Infections due to $P$. aeruginosa are seldom encountered in healthy adults;

\footnotetext{
* Correspondence: drsalamrmc@yahoo.com

${ }^{2}$ Department of Microbiology, Rajshahi Medical College, Rajshahi 6000, Bangladesh

Full list of author information is available at the end of the article
}

but in the last two decades, the organism has become increasingly recognized as the etiological agent in patients with impaired immune defenses [2]. Pseudomonads are more versatile than Enterobacteriaceae in acquiring drug resistance by various mechanisms. The production of extended-spectrum beta-lactamases (ESBLs) confers resistance at various levels to expanded spectrum Cephalosporins [3]. These enzymes are encoded by different genes located on either chromosomes or plasmids [4]. ESBLproducing bacteria may not be detectable by the routine disk diffusion susceptibility test, leading to inappropriate use of antibiotics and treatment failure.

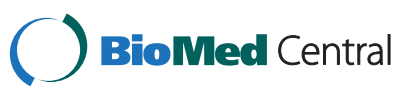


Several different methods have been suggested for the detection of ESBLs in clinical isolates [5], such as disk approximation or double disk synergy, modified double disc test (MDDT), CLSI phenotypic confirmatory method, E-test ESBL strips, three dimensional test, Vitek system, etc. While each of these tests has merit, none are able to detect all of the ESBLs encountered. Disk approximation or double disk synergy is one of the currently available and most widely used techniques for the detection of ESBLs [6].

Although bacterial resistance to the beta-lactam drugs and the mechanisms leading to this resistance have become a primary focus for clinicians and researchers, until recently, only a few studies have been carried out to detect ESBL bacteria in Bangladesh. Further, routine ESBL phenotype screening is not yet practiced in Bangladesh. The present study was conducted with an aim to detect the prevalence of ESBL-producing Pseudomonas spp. isolated from clinical samples of two tertiary care hospitals in Bangladesh.

\section{Findings \\ Materials and methods \\ Materials}

The study examined 600 swabs including from wounds ( $\mathrm{n}=200)$, pus $(\mathrm{n}=110)$, urine $(\mathrm{n}=100)$, aural $(\mathrm{n}=80)$, sputum $(\mathrm{n}=50)$, throat $(\mathrm{n}=50)$, umbilicus and conjunctiva $(\mathrm{n}=10)$ taken from patients of different ages and sex attending at Rajshahi Medical College Hospital (RMCH) and BIRDEM Hospital, Dhaka, Bangladesh from July 2000 to September 2003. Laboratory works were performed at the Microbiology laboratory of Rajshahi Medical College, BIRDEM hospital and the Molecular Biology laboratory of the Institute of Biological Sciences (IBSc), Rajshahi University. The study was ethically approved by the Ethical Review Committee of the Institute of Biological Sciences (IBSc), Rajshahi University and written informed consent was obtained from patients or a legal guardian in the case of minors.

\section{Culture and identification of species and serotypes}

Following aseptic collection, swabs were routinely inoculated onto Blood and MacConkey agar media. The plates were incubated overnight aerobically at $37^{\circ} \mathrm{C}$ and then checked for bacterial growth. Pseudomonas spp. were identified by their colony morphology, staining characters, pigment production, motility and other relevant biochemical tests as per standard methods of identification. Cetrimide agar medium was used as selective media for subculturing Pseudomonas spp. Isolates were categorized into different species based on their distinct biochemical and pigment production characteristics [7] and serotyping of Pseudomonas aeruginosa was done using commercially available (Denka Seiken Co. Ltd.,
Japan) polyvalent I, II and III group specific antisera against $14 \mathrm{O}$ antigens.

\section{Antimicrobial susceptibility testing}

Antimicrobial susceptibility testing of Pseudomonas spp. was done by the Kirby-Bauer agar diffusion method using P. aeruginosa ATCC 27853 as the control strain. Commercially available (Hi-Media) antimicrobial disks of Piperacillin (PIP $100 \mu \mathrm{g}$ ), Amikacin (AMI $30 \mu \mathrm{g}$ ), Carbinicillin (CARB $100 \mu \mathrm{g}$ ), Ceftazidime (CAZ $30 \mu \mathrm{g}$ ), Ceftriaxone (CRO $30 \mu \mathrm{g}$ ), Cefotaxime (CTX $30 \mu \mathrm{g}$ ), Tetracycline (TET $30 \mu \mathrm{g}$ ), Gentamycin (GEN $10 \mu \mathrm{g}$ ), Ciprofloxacin (CIP $5 \mu \mathrm{g}$ ), Tobramycin (TOB $10 \mu \mathrm{g}$ ), Imipenem (IMP $10 \mu \mathrm{g}$ ) and Netilmycin (NET $30 \mu \mathrm{g}$ ) were used on Mueller Hinton agar (MHA, Hi-Media) to test susceptibility. Zone of inhibition was recorded as Sensitive or Resistant according to CLSI guidelines [8].

Detection of ESBL by double disc diffusion synergy method ESBL production in Pseudomonas spp. was detected by double disk synergy test (DDST) as described by Jarlier [9]. Mueller Hinton agar was inoculated with standardized inoculum (corresponding to 0.5 McFarland tube) using sterile cotton swab. An Augmentin (20 $\mu \mathrm{g}$ Amoxicillin and $10 \mu \mathrm{g}$ of Clavulanic acid- AMC) disk was placed in the center of the plate and test disks of 3rd generation Cephalosporins (Ceftazidime- CAZ $30 \mu \mathrm{g}$, Ceftriaxone-CRO $30 \mu \mathrm{g}$, Cefotaxime-CTX $30 \mu \mathrm{g}$ ) and Aztreonam (ATM $30 \mu \mathrm{g}$ ) disks were placed at $20 \mathrm{~mm}$ distance (center to center) from the AmoxicillinClavulanic acid disk prior to incubation. The plate was incubated overnight at $35^{\circ} \mathrm{C}$. Enhancement of the zone of inhibition of any one of the four drug disks toward Amoxicillin-Clavulanic acid suggested the presence of extended-spectrum beta-lactamases.

\section{Results}

Table 1 shows the frequency distribution of Pseudomonas spp. with the number of ESBL-positive cases. Of 82 strains of Pseudomonas spp. tested for ESBL, 31 (37.8\%) were found as ESBL-positive with the highest frequency (75\%)

Table 1 Rate of detection of Pseudomonas spp. and ESBLpositive strains from clinical specimens

\begin{tabular}{ccc}
\hline Specimens (n) & Pseudomonas spp. $\mathbf{n}(\%)$ & ESBL-positive $\mathbf{n}(\%)$ \\
\hline Wound swab (200) & $19(9.5)$ & $5(26.3)$ \\
\hline Pus (110) & $20(18.2)$ & $7(35)$ \\
\hline Aural swab (80) & $18(22.5)$ & $6(33.3)$ \\
\hline Urine (100) & $12(12)$ & $9(75)$ \\
\hline Sputum (50) & $8(16)$ & $2(25)$ \\
\hline Throat swab (50) & $4(8)$ & $2(50)$ \\
\hline Others (10) & $1(10)$ & $0(0)$ \\
\hline Total & 82 & $31(37.8)$ \\
\hline
\end{tabular}


Table 2 Pseudomonas species and serotypes of Pseudomonas aeruginosa with ESBL positivity

\begin{tabular}{lcc}
\hline \multicolumn{1}{l}{ Species } & Number & ESBL-positive $\mathbf{n}(\%)$ \\
\hline $\begin{array}{l}\text { P. aeruginosa } \\
\text { Serotypes- } \mathrm{A}, \mathrm{C}, \mathrm{H}, \mathrm{I}, \mathrm{L}-25\end{array}$ & 74 & $29(39.2)$ \\
\hline $\mathrm{B}, \mathrm{J}, \mathrm{K}, \mathrm{M}-17$ & & \\
\hline $\mathrm{D}, \mathrm{E}, \mathrm{F}, \mathrm{G}, \mathrm{N}-32$ & & \\
\hline P. fluorescens & 2 & 0 \\
\hline S. multophilia & 2 & $1(50)$ \\
\hline R. pickettii & 2 & $1(50)$ \\
\hline P. alkaligenes & 2 & 0 \\
\hline Total & 82 & $31(37.8)$ \\
\hline
\end{tabular}

from urine, followed by throat (50\%), pus (35\%), aural (33.3\%) wound $(26.3 \%)$ and sputum (25\%).

Distribution of Pseudomonas species and serotypes of Pseudomonas aeruginosa with ESBL positive strains are shown in Table 2. Of 120 Pseudomonas isolates, 82 were identified at the species level based on their distinct biochemical and pigment production characteristics [7]. Pseudomonas aeruginosa, Pseudomonas fluorescens, Stenotrophomonas maltophilia, Ralstonia pickettii and Pseudomonas alkaligenes were detected at a frequency of 74 (90.2\%), 2 (2.45\%), 2 (2.45\%), 2 (2.45\%) and 2 (2.45\%) respectively. Of 74 Pseudomonas aeruginosa isolates, 29 (39.2\%) were ESBL positive. Serotyping of Pseudomonas aeruginosa revealed 25 as A,C,H,I,L (polyvalent group I), 17 as B,J,K,M (polyvalent group II) and 32 as D,E,F,G,N (polyvalent group III).

Antimicrobial susceptibility pattern revealed that Imipenem was the most effective drug against Pseudomonas spp. with susceptibility of $93.3 \%$, followed by Tobramycin (66.7\%), Amikacin (63.7\%) and Gentamycin (60\%). Susceptibility of Pseudomonas spp. to 3rd generation Cephalosporins ranged from 43.3 to $46.7 \%$ and Aminoglycosides had better efficacy than 3rd generation Cephalosporins (Table 3).

\section{Discussion}

ESBL-producing organisms pose unique challenges to clinical microbiologists, clinicians, infection control professionals and scientists engaged in finding new antibacterial molecules. ESBL-producing strains are usually found in those hospitals where antibiotic use is frequent and the patients are in critical condition. In the present study, 120 Pseudomonas spp. were isolated from 600 bacterial cultures with Pseudomonas aeruginosa as the most frequent (90.2\%) species accounting for $18 \%$ of clinical cases. The prevalence of $P$. aeruginosa is consistent with the findings of Wiblin (1997), who documented $16 \%$ prevalence for various infections [10].

The rates of ESBL-positive Pseudomonas spp. (37.8\%) and Pseudomonas aeruginosa (39.2\%) found in our study were in accordance with similar studies conducted in Bangladesh and other South East Asian countries [3,4,11-13], although low detection rates of $3.7 \%$ to $7.7 \%$ were noted in studies conducted by others $[14,15]$. Infection patterns, hospital infection control measures and antibiotic policy are all important considerations for variation of detection rates in different hospitals. Of note, we also found two ESBL-negative P. fluorescens species, and a recent study has shown that these species produce metallo-beta-lactamase, another very important betalactam inhibitor that can act as a reservoir of multidrug resistance element that may be transferred to successful $P$. aeruginosa clones [16].

The distribution of ESBL-positive Pseudomonas spp. in different samples and its resistance to 3rd generation Cephalosporins is comparable to the findings of Aggarwal et al. [4]. This resistance is due to the hydrolysis of the $\beta$-lactam ring by the action of $\beta$-lactamase enzymes. Other mechanisms for drug resistance to the $\beta$-lactam group of antibiotics are loss of outer membrane protein, production of class $\mathrm{C}$ AmpC $\beta$-lactamases and altered target sites. ESBL enzymes are inhibited by $\beta$-lactamase inhibitors, particularly Clavulanic acid and Sulbactam. Hence the use of $\beta$-lactam/ $\beta$-lactamase inhibitor combination therapy may be an alternative to 3rd generation Cephalosporins, but the effect of this combination varies depending on the subtype of ESBL present [17].

Imipenem was found to be the most efficacious drug against Pseudomonas spp. in our study, which is in accordance with findings of Ullah et al. [13]; however, notably in our study, Imipenem underperformed compared to the $100 \%$ susceptibility found in ESBL-producing gramnegative isolates, including $P$. aeruginosa, in different studies $[13,18]$. Decreased susceptibility to Imipenem is a matter of great concern for treating infections caused by Pseudomonas aeruginosa and indicates the urgent need for improved infection control strategies.

\section{Conclusion}

ESBL-producing Pseudomonas spp., and P. aeruginosa in particular, were found to be frequent isolates from two tertiary care hospitals in Bangladesh with limited susceptibility

Table 3 Antimicrobial susceptibility pattern of Pseudomonas spp

\begin{tabular}{|c|c|c|c|c|c|c|c|c|c|c|c|c|}
\hline \multicolumn{13}{|c|}{ Antimicrobial drugs } \\
\hline Susceptibility\% & PIP & CARB & CAZ & CRO & CTX & CIP & TET & $\mathrm{AMI}$ & GEN & TOB & NET & IMP \\
\hline & 58 & 43.3 & 46.7 & 46.4 & 43.3 & 40 & 32.5 & 63.7 & 60 & 66.7 & 53.3 & 93.3 \\
\hline
\end{tabular}


to antimicrobials. Further, decreased susceptibility to Imipenem is a matter of great concern as it is the drug of choice in the treatment of Pseudomonad infection.

\section{Competing interests}

The authors declare that they have no competing interests.

\section{Authors' contributions}

SB conceived the research idea and performed the laboratory work. KFA and NB helped in sample collection and preparation of the manuscript. JAH and $\mathrm{PH}$ helped in designing the study and supervision of the work. MAS contributed intellectual thought, final revision and editing of the manuscript. All authors have read and approved the submitted version of manuscript.

\section{Acknowledgement}

We acknowledge Colgate Ross MPH, Research Specialist, University of Vermont College of Medicine, Infectious Disease Unit, Burlington, VT 05405, USA, for her contribution in language correction of the manuscript.

\section{Author details}

'Department of Microbiology, Green Life Medical College, Dhaka, Bangladesh. ${ }^{2}$ Department of Microbiology, Rajshahi Medical College, Rajshahi 6000, Bangladesh. ${ }^{3}$ Department of Microbiology, Pabna Medical College, Pabna, Bangladesh. ${ }^{4}$ Institute of Biological Sciences, University of Rajshahi, Rajshahi 6205, Bangladesh. ${ }^{5}$ Department of Microbiology, Ibrahim Medical College, Dhaka 1000, Bangladesh.

Received: 9 September 2012 Accepted: 4 January 2013 Published: 5 January 2013

\section{References}

1. Dundar D, Otkun M: In-vitro efficacy of synergistic antibiotic combinations in multidrug resistant pseudomonas aeruginosa strains. Yonsei Med J 2010, 51:111-116.

2. Wirth FW, Picoli SU, Cantarelli W, Gonçalves AL, Brust FR, Santos LM, Barreto MF: Metallo- $\beta$-lactamase-producing Pseudomonas aeruginosa in two hospitals from Southern Brazil. Braz J Infect Dis 2009, 13:170-172.

3. Mirsalehian A, Feizabadi M, Nakhjavani FA, Jabalameli F, Goli H, Kalantari N: Detection of VEB-1, OXA-10 and PER-1 genotypes in extended-spectrum beta-lactamase-producing Pseudomonas aeruginosa strains isolated from burn patients. Burns 2010, 36:70-74.

4. Aggarwal R, Chaudhary U, Bala K: Detection of extendedspectrum $\beta$ - lactamase in Pseudomonas aeruginosa. Indian J Pathol Microbiol 2008, 51:222-224

5. Drieux L, Brossier F, Sougakoff W, Jarlier V: Phenotypic detection of extended-spectrum beta-lactamase production in Enterobacteriaceae: review and bench guide. Clin Microbiol Infect 2008, 14(Suppl 1):90-103.

6. Kader AA, Angamuthu KK, Kamath KA, Zaman MN: Modified double-disc test for detection of extended-spectrum [beta]-lactamases in Escherichia coli and Klebsiella pneumoniae. British J Biomedical Science 2006, 63(2):51-54.

7. Palleroni NJ: Pseudomonadaceae. In Bergey's Manual of Systematic Bacteriology. Edited by Krieg NR, Holt JG. Baltimore: Williams \& Wilkins; 1984:141-219.

8. National Committee for Clinical Laboratory Standards: Performance standards for antimicrobial disk susceptibility tests. Twenty-First editionth edition. Wayne, PA, USA: Informational Supplement M100-S21. CLSI; 2010.

9. Jarlier $V$, Nicolas MH, Fournier G, Philippon A: Extended spectrum $\beta$-lactamases conferring transferable resistance to newer $\beta$ - lactam agents in Enterobacteriaceae: Hospital prevalence and susceptibility patterns. Rev Infect Dis 1988, 10:867-878.

10. Wiblin RT: Nosocomial pneumonia. In Prevention and control of nosocomial infections. 3rd edition. Edited by Wenzel RP. Baltimore: Williams and Wilkins; 1997:807-819.

11. Pandey A, Malenie R, Asthana AK: Beta-lactamase producing Pseudomonas aeruginosa in hospitalised patients. Indian J Pathol Microbiol 2005, 48(4):530-533.

12. Haque R, Salam MA: Detection of ESBL producing nosocomial gram negative bacteria from a tertiary care hospital in Bangladesh. Pak J Med Sci 2010, 26(4):887-891.
13. Ullah F, Malik SA, Ahmed J: Antimicrobial susceptibility and ESBL prevalence in pseudomonas aeruginosa isolated from burn patients in the North West of Pakistan. Burns 2009, 35(7):1020-1025.

14. Woodford N, Zhang J, Kaufmann ME, Yarde S, Tomas Mdel M, Faris C, Vardhan MS, Dawson S, Cotterill SL, Livermore DM: Detection of Pseudomonas aeruginosa isolates producing VEB-type extendedspectrum $\beta$-lactamases in the United Kingdom. J Antimicrob Chemother 2008, 62:1265-1268.

15. Lim KT, Yasin RM, Yeo CC, Puthucheary SD, Balan G, Maning N, Wahab ZA, Ismail N, Tan EA, Mustaffa A, Thong KL: Genetic fingerprinting and antimicrobial susceptibility profiles of Pseudomonas aeroginosa hospital isolates in Malaysia. J Microbiol Immunol Infect 2009, 42:197-209.

16. Juan C, Zamorano L, Mena A, Albertí S, Pérez JL, Oliver A: Metallo-beta-lactamase-producing pseudomonas putida as a reservoir of multidrug resistance elements that can be transferred to successful pseudomonas aeruginosa clones. J Antimicrob Chemother 2010, 65(3):474-478

17. Nathisuwan S, Burgess DS, Lewis JS: 2nd Extended spectrum $\beta$-lactamases: epidemiology, detection and treatment. Pharmacotherapy 2001, 21:920-928

18. Winokur PL, Canton R, Casellas JM, Legakis N: Variations in the prevalence of strains expressing an extended spectrum $\beta$-lactamase phenotype and characterisation of isolates from Europe, the Americans and the Western Pacific region. Clin Infect Dis 2001, 32:594-603.

doi:10.1186/1756-0500-6-7

Cite this article as: Begum et al:: Detection of extended spectrum $\beta$ lactamase in Pseudomonas spp. isolated from two tertiary care hospitals in Bangladesh. BMC Research Notes 2013 6:7.

\section{Submit your next manuscript to BioMed Central and take full advantage of:}

- Convenient online submission

- Thorough peer review

- No space constraints or color figure charges

- Immediate publication on acceptance

- Inclusion in PubMed, CAS, Scopus and Google Scholar

- Research which is freely available for redistribution 